\title{
Prevalência de lesão por pressão em um hospital geral
}

\author{
Prevalence of pressure injury a general hospital \\ Prevalencia de lesiones por presión en un hospital general
}

Sandra Regina Silva de Moura, ${ }^{1,2 *}$, Daniela Pinheiro de Lima Melo ${ }^{1}$, Gabriela Maria da Silva Rocha ${ }^{2}$, Éricka Roberta Conceição da $\mathrm{Cruz}^{3}$.

\section{RESUMO}

Objetivo: Identificar a prevalência de lesão por pressão em um hospital geral e verificar o perfil clínico epidemiológico dos pacientes atendidos. Métodos: Foi realizado um estudo quantitativo, tipo transversal, onde foram analisados todos os casos de lesão por pressão notificada à comissão de curativos, no período de janeiro de 2017 a dezembro de 2018, através de frequências absolutas e relativas. Resultados: A prevalência das lesões por pressão foi de 1,2\%, distribuídas igualmente entre ambos os sexos, a faixa etária mais prevalente foi nos pacientes $>60$ anos $(45,9 \%)$, a principal causa de internamento foram as neoplasias $(15,6 \%)$, as enfermarias de adultos tiveram $55,1 \%$ dos casos, a região mais acometida foi a sacral com $66,2 \%$, e $21,7 \%$ das lesões se encontravam no estágio II. Considerações finais: Os resultados apontaram que do ano de 2017 para 2018 ocorreu uma diminuição das LPP, mais que deve ser mantido o monitoramento deste indicador para que venha proporcionar maior nível de segurança aos pacientes e ajudar na redução de custos relacionados a eventos adversos que são altamente evitáveis pelas instituições.

Palavras-chave: Enfermagem, Segurança do paciente, Úlcera por pressão.

\begin{abstract}
Objective: To identify the prevalence of pressure injuries in a general hospital and to verify the epidemiological clinical profile of the patients seen. Methods: A quantitative, cross-sectional study was carried out, in which all cases of pressure injuries notified to the dressing committee were analyzed, from January 2017 to December 2018, using absolute and relative frequencies. Results: The prevalence of pressure injuries was $1.2 \%$, distributed equally between both sexes, the most prevalent age group was in patients $>60$ years $(45.9 \%)$, the main cause of hospitalization were neoplasms (15,6\%), adult wards had $55.1 \%$ of cases, the most affected region was the sacral region with $66.2 \%$, and $21.7 \%$ of the injuries were in stage II. Final considerations: The results showed that from 2017 to 2018 there was a decrease in LPP, more that the monitoring of this indicator should be maintained so that it will provide a higher level of safety to patients and help in reducing costs related to adverse events that are highly avoidable by the institutions.
\end{abstract}

Keywords: Nursing, Patient safety, Pressure ulcer.

\section{RESUMEN}

Objetivo: Identificar la prevalencia de lesiones por presión en un hospital general y verificar el perfil clínico epidemiológico de los pacientes atendidos. Métodos: Se realizó un estudio cuantitativo, transversal, en el que

\footnotetext{
${ }^{1}$ Faculdade Pernambucana de Saúde (FPS), Recife - PE. *E-mail: sandra.moura@fps.edu.br

2 Instituto de Medicina Integral Prof. Fernando Figueira (IMIP), Recife - PE.

${ }^{3}$ Secretaria Estadual de Saúde de Pernambuco (SES/PE), Recife - PE.
} 
se analizaron todos los casos de lesiones por presión notificadas al comité de vestuario, desde enero de 2017 hasta diciembre de 2018, utilizando frecuencias absolutas y relativas. Resultados: La prevalencia de lesiones por presión fue de $1,2 \%$, distribuida equitativamente entre ambos sexos, el grupo de edad más prevalente fue en pacientes> 60 años $(45,9 \%)$, la principal causa de hospitalización fueron las neoplasias (15,6\%), las salas de adultos presentaron el $55,1 \%$ de los casos, la región más afectada fue la región sacra con el $66,2 \%$ y el $21,7 \%$ de las lesiones estaban en estadio II. Consideraciones finales: Los resultados arrojaron que de 2017 a 2018 hubo una disminución en la LPP, más que se debe mantener el seguimiento de este indicador para que brinde un mayor nivel de seguridad a los pacientes y ayude en la reducción de costos relacionados con eventos adversos que son altamente evitable por las instituciones.

Palabras clave: Enfermería, Seguridad del paciente, Úlcera por pressión.

\section{INTRODUÇÃO}

Segundo a Organização Mundial da Saúde (OMS), o conceito de Segurança do Paciente refere-se à redução dos riscos de danos associados à assistência em saúde e visa minimizar esses danos a um mínimo aceitável, incentivando as instituições de saúde em todo o mundo a realizarem ações em prol da adesão as boas práticas e ao cuidado seguro ao paciente. Considerando as lesões por pressão (LPP), um tipo de eventos adversos (EA), ou seja, um incidente ou circunstância não intencional que gerou o comprometimento da saúde física ou psicossocial do paciente, as LPP aumentam o tempo de permanência e o custo do internamento do paciente. Diminuem o bem-estar dos pacientes, tornando-se imprescindível reforçar os esforços na implementação de medidas de prevenção efetivas e tratamento oportuno das lesões já estabelecidas (WHO, 2010; SIMAN AG e BRITO MJM, 2019; SOUSA NCR, 2017; MEIRELES VC e BALDISSERA VDA, 2019).

Entendendo as LPP, como uma ocorrência decorrente, na maioria dos casos, da não adoção de medidas preventivas, como a mudança de decúbito, manutenção da pele hidratada e sem umidade, uso de coxins e coberturas preventivas, entre outras. A prevalência das LPP se constitui como um indicador negativo da qualidade dos cuidados prestados, sendo avaliado internacionalmente como um EA, especialmente por 95,0\% serem consideradas potencialmente evitáveis, ou seja, que poderiam ser prevenidas com a adesão as medidas de prevenção (SOUSA NCR, 2017).

Diante da magnitude dos danos gerados nos pacientes, por falhas na assistência, a OMS, institui a World Alliance for Patient Safety, que com base nas causas que mais geravam danos aos pacientes estabeleceram as Metas Internacionais para Segurança do Paciente, a fim de promover a adesão a práticas para minimização de danos aos pacientes, são elas: 1 - identificar corretamente o paciente; 2 - melhorar a comunicação entre profissionais de saúde; 3 - melhorar a segurança na prescrição, no uso e administração de medicamentos; 4 - assegurar cirurgia em local de intervenção, procedimento e paciente correto; 5 - higienizar as mãos para evitar infecções; 6 - reduzir o risco de quedas e úlceras por pressão (BRASIL, 2013).

Com a finalidade de colaborar para a qualificação do cuidado em todos os estabelecimentos de saúde no Brasil e estimular as discussões sobre o tema e implementá-lo na formação profissional, foi instituído o Programa Nacional de Segurança do Paciente (PNSP), pela Portaria ํㅜ 529/2013 do Ministério da Saúde e regulamentado pela Resolução da Diretória Colegiada (RDC) no 36/2013 da Agência Nacional de Vigilância Sanitária (Anvisa). O programa institui, entre outras demandas, a criação de 6 protocolos básicos de segurança do paciente, baseados nas metas internacionais, entre eles o protocolo de prevenção de úlcera por pressão, antiga nomenclatura das LPP (BRASIL, 2013).

Aprovado pela Portaria $n^{\circ}$. 1.377, de 9 de julho de 2013, que detalha estratégias importantes para redução das LPP no ambiente hospitalar, como a realização da avaliação de risco do paciente para desenvolvimento de LPP, a inspeção diária da pele e a mudança de decúbito. Além de incluir outros profissionais, além da 
enfermagem, na prevenção desses episódios, como os nutricionistas, destacando a importância da equipe multiprofissional (BRASIL, 2013).

A LPP é um evento adverso que se configura como uma das diversas complicações às quais pacientes hospitalizados estão suscetíveis, podendo levar à destruição parcial ou total dos tecidos. As LPP são definidas pelo National Pressure Ulcer Advisory Panel (NPUAP) como um dano localizado na pele e/ou tecidos moles subjacentes, geralmente sobre uma proeminência óssea ou relacionada ao uso de dispositivo hospitalar.

Em 2016, foi modificada a terminologia de Úlcera por Pressão para Lesão por Pressão, por essas lesões apresentarem-se em pele íntegra ou como úlcera, alterando ainda os parâmetros para sua classificação. Esse tipo de lesão acontece como resultado da pressão prolongada, da pele sobre proeminências ósseas, combinada ou não com o cisalhamento. A resistência da pele à pressão e ao cisalhamento pode ser diminuída por fatores como, a nutrição, a perfusão, as comorbidades e pelo estado de gravidade do paciente (SOBEST, 2016).

Segundo as novas definições do NPUAP, as LPP são classificadas em estágios, numerados de 1 a 4 e em lesões com estágio não classificável e em tissular profunda. Onde as lesões estágio 1 possuem pele íntegra e eritema não branqueável; no estágio 2 apresentam perda de pele parcial, com exposição da derme; nas de estágio 3 a perda de pele é em sua espessura total; no estágio 4 as lesões apresentam perda tissular; as LPP com perda de pele na sua espessura total e perda tissular não visível são categorizadas como não classificável e as lesões que apresentam descoloração do vermelho escuro a púrpura persistente e não branqueável são classisficadas como tissular profunda (SOBEST, 2016).

A taxa de incidência e prevalência descritas na literatura apresenta variações que se devem às características dos pacientes e ao nível de assistência ofertada em cada unidade, diferenciando-se em cuidados de longa permanência, cuidados agudos e atenção domiciliar.

A prevalência de LPP em hospitais é de 15,0\% e a incidência é de 7,0\%. No Reino Unido, casos novos de LPP acometem de $4,0 \%$ a $10,0 \%$ dos pacientes admitidos nos hospitais. Já no Brasil, embora não existam trabalhos sobre a incidência e prevalência de LPP, em âmbito nacional, a prevalência varia entre 5,3\% e 26,0\% (MELLEIRO MM, 2015; GALVÃO NS, et al., 2015; CARVALHO F, 2019).

Os enfermeiros na condição de líder, tem se responsabilizado por prever e prover recursos humanos, materiais e estruturais, fundamentando-se em evidências cientificas, para implantar medidas preventivas para as LPP, e quando o desenvolvimento de LPP é inevitável, torna-se necessária a adoção de ações terapêuticas adequadas a fim de minimizar as suas conseqüências e evitar a evolução da sua gravidade (SILVA DRA, 2017). Atualmente as LPP representam um importante problema de saúde pública, sendo apontada como um indicador de qualidade da assistência, necessitando de maiores esforços na análise de sua ocorrência (PEREIRA AFM, 2017).

Diante da relevância dessa temática para melhoria da qualidade da assistência, especialmente dos cuidados de enfermagem e da necessidade de verificar a efetividade das medidas preventivas, objetivou-se com esse estudo contribuir o maior conhecimento acerca dessa realidade nos serviços de saúde e consequentemente subsidiar a adoção de medidas especializadas, oportunas e direcionadas para a qualificação da assistência ofertada aos pacientes.

\section{MÉTODOS}

A população do estudo foi composta pelos pacientes hospitalizados em munícipio do estado do Pernambuco, que desenvolveram LPP durante o período de internação. Se realizou um estudo do tipo transversal, retrospectivo, descritivo e de natureza quantitativa. Possibilitando explorar a distribuição das LPP e as características da população estudada. O estudo ocorreu no período de janeiro/2017 a Agosto/2018.

O Hospital Escola, entidade 100,0\% filantrópico, que atua nas áreas de assistência médico-social, ensino, pesquisa e extensão comunitária. Com 1.171 leitos, sendo 101 de Unidade de Terapia Intensiva (UTI) e, além disso, realiza mais de 700 mil atendimentos anuais em seus serviços. Atende todas as especialidades 
médicas, pediatria, ginecologia, clínica médica e cirúrgica, hemodiálise, oncologia, cardiologia, neurologia e realiza transplantes.

Foram incluídos na pesquisa todos os pacientes internados que foram notificados com LPP, no período de Janeiro/2017 a Dezembro/2018. Nesse estudo, foram utilizados dados secundários, coletados a partir do Sistema de Notificação da Vigilância Sanitária (Notivisa), implantado na Gerência de Risco da instituição e alimentado pelas Notificações de EA, pelos registros de atendimento da Comissão de Curativos e pelas solicitações de liberação de coberturas especiais para tratamento das lesões. Os dados foram processados e analisados pelas pesquisadoras, através do software Excel versão 2016 onde foi realizada a análise descritiva dos dados e calculadas as frequências relativas e absolutas.

Este estudo respeitou os padrões éticos preconizados pela Resolução 466/12, foi aprovado pelo Comitê de Ética da Faculdade Pernambucana de Saúde (CEP/FPS) sob CAAE: 67031017.4.0000.5569. Durante a submissão foi solicitada a dispensa do Termo de Consentimento Livre e Esclarecido (TCLE), que foi aceita, tendo em vista que se trata de um estudo que utilizou dados secundários.

\section{RESULTADOS}

A amostra do estudo foi composta por 1.234 LPP em pacientes hospitalizados. Foram consideradas as lesões que se desenvolveram na instituição e as pré-existentes na admissão do paciente na instituição, sendo 680 notificadas no ano de 2017 e 554 no ano de 2018 . A prevalência do período total do estudo foi de $1,2 \%$ e, $1,4 \%$ em 2017 e de $1,0 \%$ em 2018.

Analisando o perfil dos pacientes acometidos com LPP, o sexo masculino constituiu o maior percentual, $53,00 \%$ em 2017 e o sexo feminino 53,97\% no ano de 2018, no somatório dos anos, o sexo feminino representou 50,08\% das LPP.A faixa etária com maior prevalência foi igual nos dois anos, pacientes acima de 60 anos.

Em 2017 com 38,97\% e em 2018 com 54,51\%, seguidos pelos pacientes de 19 aos 59 anos em 2017 com $35,44 \%$ e em $2018 \mathrm{com} 27,98 \%$. No total do período as faixas etárias mais atingidas foram iguais $>60$ anos $(45,95 \%)$ e dos 19 aos 59 anos $(32,09)$.

Entre os setores com maior prevalência de pacientes com LPP nos dois anos estão as enfermarias de adulto, 49,41 em 2017 e 62, 09 em 2018, seguida pela unidade de terapia intensiva que também nos dois anos estavam com 46,32\% em 2017 e no ano de 2018 com 36,64\%. Com relação ao motivo da internação, essa variável obteve $56,8 \%$ de ignorabilidade em 2017 e 45,9\% em 2018, uma fragilidade no registro dos dados.

Em seguida o diagnóstico encontrado na pesquisa em ambos os anos foram as neoplasias com 11,9\% em 2017 e 20,2\% em 2018, seguidos pela variável outros diagnósticos com 10,2\% em 2017 e em 2018 com $11,2 \%$, neste item foram incluídos diagnósticos não classificáveis nas categorias pré-definidas e com baixa incidência. No período total do estudo as neoplasias representaram $15,68 \%$ do diagnóstico de internação dos pacientes que desenvolveram as lesões, seguidos de outros diagnósticos com 10,86\% e as doenças do sistema neurológico 6,89\% (Tabela 1).

A localização anatômica mais prevalente nos dois anos foram as lesões em região sacral com $62,35 \%$ em 2017 e $71,12 \%$ em 2018, total $66,29 \%$. Seguidos pelos membros inferiores que ficaram com $9,85 \%$ e com $9,03 \%$, nos anos de 2017 e 2018, respectivamente. E as lesões na região trocantérica, $6,18 \%$ em 2017 e 7 , $40 \%$ em 2018. No total a região sacral ficou com $66,29 \%$ das lesões, os membros inferiores $9,48 \%$ e a região trocantérica $6,73 \%$. Com relação aos estágios que se encontravam as lesões no momento da notificação, foi identificado um percentual de $48,62 \%$ de ignorabilidade no somatório dos anos. Seguindo, os dois anos foram a de estágio II com 15,1\% em 2017 e 29,7\% em 2018, seguidos em 2017 pelas lesões de estágio I com 11,0\% e em 2018 a de estágio III com 28,3\%. Um dado importante é que no total do período estudado, apenas 3,00\% das lesões foram notificadas no estágio IV, as demais foram notificadas mais precocemente (Tabela 2). 
Tabela 1 - Distribuição dos pacientes com LPP por sexo, idade, setor de internação e diagnóstico, $n=1254$. 2017 e 2018.

\begin{tabular}{|c|c|c|c|c|c|c|}
\hline \multirow[t]{2}{*}{ Variáveis } & \multicolumn{2}{|c|}{$\begin{array}{c}\text { Ano } 2017 \\
\text { (n 680) }\end{array}$} & \multicolumn{2}{|c|}{$\begin{array}{c}\text { Ano } 2018 \\
\text { (n 554) }\end{array}$} & \multicolumn{2}{|c|}{$\begin{array}{c}\text { Total } \\
\text { (n 1234) }\end{array}$} \\
\hline & $\mathbf{n}$ & $\%$ & $\mathbf{N}$ & $\%$ & $\mathbf{n}$ & $\%$ \\
\hline \multicolumn{7}{|c|}{ Sexo } \\
\hline Feminino & 319 & 47,00 & 299 & 53,97 & 618 & 50,08 \\
\hline Masculino & 361 & 53,00 & 255 & 46,03 & 616 & 49,92 \\
\hline \multicolumn{7}{|c|}{ Idade } \\
\hline $0-9$ Anos & 94 & 13,82 & 19 & 3,43 & 113 & 9,16 \\
\hline $10-18$ Anos & 46 & 6,76 & 23 & 4,15 & 69 & 5,59 \\
\hline 19 - 59 Anos & 241 & 35,44 & 155 & 27,98 & 396 & 32,09 \\
\hline Mais De 60 Anos & 265 & 38,97 & 302 & 54,51 & 567 & 45,95 \\
\hline Ignorado & 34 & 5,00 & 55 & 9,93 & 89 & 7,21 \\
\hline \multicolumn{7}{|c|}{ Setor de Internação } \\
\hline Enfermaria Adulto & 336 & 49,41 & 344 & 62,09 & 680 & 55,11 \\
\hline Unidade de Tratamento Intensivo & 315 & 46,32 & 203 & 36,64 & 518 & 41,98 \\
\hline Pediatria & 22 & 3,24 & 6 & 1,08 & 28 & 2,27 \\
\hline Centro de Atenção à Mulher & 7 & 1,03 & - & - & 7 & 0,57 \\
\hline Ignorado & - & - & 1 & 0,18 & 1 & 0,08 \\
\hline \multicolumn{7}{|c|}{ Diagnóstico } \\
\hline Ignorado & 386 & 56,76 & 254 & 45,85 & 640 & 51,86 \\
\hline Neoplasia & 81 & 11,91 & 112 & 20,22 & 193 & 15,64 \\
\hline Outros Diagnósticos & 72 & 10,59 & 62 & 11,19 & 134 & 10,86 \\
\hline Doenças do Sistema Neurológico & 69 & 10,15 & 16 & 2,89 & 85 & 6,89 \\
\hline Doenças do Sistema Respiratório & 20 & 2,94 & 50 & 9,03 & 70 & 5,67 \\
\hline Doenças do Sistema Cardiovascular & 19 & 2,79 & 33 & 5,96 & 52 & 4,21 \\
\hline Transplantes & 22 & 3,24 & 13 & 2,35 & 35 & 2,84 \\
\hline Doenças do Sistema Geniturinário & 11 & 1,62 & 14 & 2,53 & 25 & 2,03 \\
\hline
\end{tabular}

Fonte: Moura SRS, et al., 2020; dados extraídos do Notivisa.

Tabela 2 - Distribuição de frequência por localização anatômica da lesão e estágio, n=1234. 2017 e 2018.

\begin{tabular}{|c|c|c|c|c|c|c|}
\hline \multirow[t]{2}{*}{ Variáveis } & \multicolumn{2}{|c|}{$\begin{array}{c}\text { Ano } 2017 \\
\text { (N 680) }\end{array}$} & \multicolumn{2}{|c|}{$\begin{array}{c}\text { Ano } 2018 \\
\text { (N 554) }\end{array}$} & \multicolumn{2}{|c|}{$\begin{array}{c}\text { Total } \\
\text { (N 1234) }\end{array}$} \\
\hline & $\mathbf{N}$ & $\%$ & $\mathbf{N}$ & $\%$ & $\mathbf{N}$ & $\%$ \\
\hline \multicolumn{7}{|c|}{ Localização anatômica } \\
\hline Sacral & 424 & 62,35 & 394 & 71,12 & 818 & 66,29 \\
\hline Membros Inferiores & 67 & 9,85 & 50 & 9,03 & 117 & 9,48 \\
\hline Trocantéricas & 42 & 6,18 & 41 & 7,40 & 83 & 6,73 \\
\hline Calcâneo & 39 & 5,74 & 32 & 5,78 & 71 & 5,75 \\
\hline Ignorado & 39 & 5,74 & 10 & 1,81 & 49 & 3,97 \\
\hline Escapular & 19 & 2,79 & 8 & 1,44 & 27 & 2,19 \\
\hline Membros Superiores & 19 & 2,79 & 8 & 1,44 & 27 & 2,19 \\
\hline Septo nasal & 16 & 2,35 & - & - & 16 & 1,30 \\
\hline Isquiática & 3 & 0,44 & 6 & 1,08 & 9 & 0,73 \\
\hline Múltiplas lesões & 5 & 0,74 & 2 & 0,36 & 7 & 0,57 \\
\hline Occipital & 6 & 0,88 & 1 & 0,18 & 7 & 0,57 \\
\hline Temporal & 1 & 0,15 & 2 & 0,36 & 3 & 0,24 \\
\hline \multicolumn{7}{|c|}{ Estágio } \\
\hline Ignorado & 430 & 63,24 & 170 & 30,69 & 600 & 48,62 \\
\hline Estágio II & 103 & 15,15 & 165 & 29,78 & 268 & 21,72 \\
\hline Estágio III & 54 & 7,94 & 157 & 28,34 & 211 & 17,10 \\
\hline Estágio I & 75 & 11,03 & 34 & 6,14 & 109 & 8,83 \\
\hline Estágio IV & 16 & 2,35 & 21 & 3,79 & 37 & 3,00 \\
\hline Não classificável & 2 & 0,29 & 7 & 1,26 & 9 & 0,73 \\
\hline
\end{tabular}

Fonte: Moura SRS, et al., 2020; dados extraídos do Notivisa.

O desbridamento cirúrgico, que é um procedimento realizado apenas mediante a necessidade, foi realizado em 1,15\% dos pacientes em 2017 e 2,17\% em 2018. Nos demais pacientes a realização desse 
procedimento não foi necessária, demonstrando que a realização de curativos oportuna, com coberturas que realizam desbridamento enzimático foi eficaz, ou a lesão não evoluiu ao ponto de apresentar tecido necrótico.

\section{DISCUSSÃO}

Ao comparar a prevalência de LPP encontrada nesse estudo com outros, percebemos que o indicador levantado se encontra abaixo da prevalência nacional e internacional, 39,8\% e 15,0\% respectivamente, diferenças nos métodos utilizados nesses estudos justificam as diferenças de valores. Outro viés é o perfil dos pacientes atendidos na instituição estudada, onde considerou-se todo o paciente hospitalizado, como em risco para LPP (BRASIL, 2013).

A análise desde estudo demonstra que não existe diferença entre os gêneros, para o desenvolvimento das lesões, apesar das diferenças na quantidade dos tecidos musculares e adiposas, entre os sexos. A amostra trouxe $50,1 \%$ de pacientes do sexo feminino e $49,9 \%$ do sexo masculino, a diferença foi de 2 pacientes a mais em relação ao outro sexo, semelhante a um estudo realizado por Melo $E$ (2018) em um hospital de atenção secundária dentro da rede pública de saúde do Ceará, onde 51,5\% dos pacientes acometidos com LPP eram do sexo masculino e 48,5\% do feminino. Outros estudos, como Costa A (2018) e Santos LRO (2016) apontam maior frequência das lesões em pacientes do sexo masculino.

Assim como descrito por Teixeira AKS, et al. (2017) a faixa etária mais acometida pelas LPP, são os pacientes acima dos 60 anos, sendo assim a idade surge como um fator contribuinte para o desenvolvimento dessas lesões, pois o envelhecimento retarda o processo de cicatricial, prejudica a vascularização, torna a pele mais sensível e diminui as camadas de tecido muscular e adiposo.

As evidências científicas apontam as UTIs como à unidade com maior prevalência de LPP, o que se justifica pelo estado de maior gravidade dos pacientes e em decorrência disso, a maior frequência de pacientes com a restrição de mobilidade, uso de dispositivos hospitalares, umidade da pele (TEXEIRA AKS, et al., 2017).

Em nosso estudo a maior prevalência de LPP foi nas enfermarias adulto, em decorrência do maior número desses leitos na instituição, seguido das UTIs, assim como foi identificado maior número de LPP em enfermarias. Este achado sinaliza a necessidade de investir em capacitações dos profissionais envolvidos na assistência aos pacientes desses setores, além de ações de supervisão e incentivo a prevenção desse EA (MEDEIROS LNB, et al., 2017).

Assim como no estudo de Costa A, et al. (2018), o diagnóstico mais prevalente como causa de internação dos pacientes que desenvolveram LPP, estava relacionado ao perfil de internações da instituição, na instituição estudada os pacientes com doenças neoplásicas são significativos no perfil de atendimento, refletindo proporcionalmente nesta variável.

Já em relação à localização anatômica, a principal localização acometida, as lesões na região sacral com cerca de $55,5 \%$ a $66,7 \%$ de prevalência nessa região, considerada uma das mais suscetíveis ao desenvolvimento de LPP em razão da extensa proeminência ósseas, baixa densidade de tecido na região, proximidade com áreas de incontinência, que favorecem a umidade da pele e ser uma área com bastante atrito durante o manuseio do paciente no leito (PETZ FFC, et al., 2017; MELO E, et al., 2018).

O estágio da lesão com maior acometimento foi o estágio II com $21,7 \%$, o que é igualmente referido em outras pesquisas (MELO E, et al., 2018; TEIXEIRA AKS, et al., 2017; PETZ F et al., 2017). Este estágio de lesão geralmente resultante de microclima inadequado e cisalhamento da pele na região da pélvis e no calcâneo (SOBEST, 2016).

Em algumas lesões com agravamento foi necessário a realização do procedimento de desbridamento cirúrgico, que consiste na remoção de tecidos mortos, desvitalizados ou contaminados, assim como qualquer corpo estranho no leito da ferida, ajudando a reduzir o número de microrganismos, toxinas e outras 
substâncias que inibem a cicatrização. Esse procedimento foi necessário em apenas 1,6\% dos pacientes (SANTOS ICRV, et al., 2016).

As LPP são avaliadas internacionalmente como um significativo EA e consequentemente representando um relevante desafio para os estabelecimentos de saúde, que necessitam investir esforços na melhoria contínua dos cuidados seguros em saúde.

Esse tipo de dano representa um aumento potencial do tempo de internação, dos custos do tratamento, da morbidade, da mortalidade hospitalar e atinge um elevado número de pacientes (BERNARDES RM e CALIRI MHL, 2016).

A equipe de enfermagem, em especial os enfermeiros, devem se apropriar de conhecimento sobre as estratégias de prevenção e opções de tratamento, fornecendo assim embasamento para minimizar esse dano ao paciente. Apesar da equipe de enfermagem ser agente ativo, os demais membros da equipe multidisciplinar devem contribuir no cuidado a esses pacientes.

Esse tipo de EA ainda é muito evidente nos serviços de saúde em decorrência da assistência ser ofertada de maneira aleatória, sem adesão a protocolos baseados nas melhores evidências científicas, falta de atualização dos profissionais e falta de implantação de estratégias conhecidamente eficazes (DANTAS ALM, 2019).

A estratégia para minimização desse significativo EA tem potencial para reduzir os custos hospitalares, reduzir o desgaste físico e emocional da equipe que presta assistência, aos pacientes e aos familiares, favorecendo o bem-estar de todos esses atores e diminuindo os danos causados pelas LPP (CARVALHO F, 2019).

O público mais prevalente em nosso estudo é reconhecido em outros estudos, como mais propenso a adquirirem esse tipo de lesão, pelo estado de gravidade, dificuldade e restrições na mobilidade, e diminuição do processo de cicatrização da pele. Constatamos ainda que as lesões mais prevalentes são de fácil detecção e tratamento, por se tratar de estágios iniciais, portanto identificado a LPP oportunamente e adotado tratamento condizente com a necessidade, possibilita se a redução do dano ao paciente, melhorando a assistência e seu bem-estar (MELO E, et al., 2018; TEIXEIRA AKS, et al., 2017; PETZ F et al., 2017).

Segundo Girondi J, et al. (2020) a fim de melhorar a segurança do paciente, as instituições devem atentar para o manejo correto do estado nutricional dos pacientes, hidratação da pele, controle do microclima e adoção de estratégias para minimização da pressão nas áreas de proeminências ósseas e insperção diária da pele, como principais precauções para prevenção do surgimento de LPP. Além desses fatores, Martins NBM, et al. (2020) alerta para os cuidados com a fixação de dispositivos hospitalares e adesivos na pele dos pacientes como potenciadores para o desenvolvimento de LPP.

Os dados foram comparados na maioria dos casos com artigos de estudos nas unidades de terapia intensiva, sendo um limitador, devido à escassez de estudos que abordem a prevalência nos hospitais como um toso, já que o estudo mostra a prevalência do hospital e não só de um setor específico. Outro limitador do estudo foram as variáveis omissas ou ignoradas, nas notificações de EA e no prontuário do paciente.

\section{CONSIDERAÇÕES FINAIS}

Constatamos que os pacientes com idade avançada e doenças que necessitam de longo período de internação, apresentam maior risco para o desenvolvimento de LPP, necessitando de maior atenção na implementação e manutenção das medidas de prevenção. A fim de reduzir este agravo, recomenda-se que práticas de prevenção de LPP sejam ampliadas na assistência ao paciente, que os profissionais sejam sensibilizados sobre a relevância da adesão as boas práticas e que estratégias como o protocolo de prevenção a LPP sejam realizadas afinco. Sugere-se, como contribuição para instituição, a melhoria da comunicação, com melhor preenchimento das notificações de EA e registros das LPP nos prontuários. Outra 
sugestão é a realização de estudos em hospitais gerais, uma vez que a maioria dos estudos sobre LPP são restritos as UTI.

\section{AGRADECIMENTOS}

Agradecemos a instituição pela anuência para realização do estudo e aos profissionais dos setores envolvidos na coleta de dados pela recepção, disponibilidade e apoio.

\section{REFERÊNCIAS}

1. BERNARDES RM, CALIRI MHL. Prevalência de úlcera por pressão em hospital de emergência: estudo transversal. Revista Estima, 2016; 14(2):99.

2. BRASIL. Portaria n. 1.377, de 9 de julho de 2013. Aprova o protocolo para Prevenção de Úlcera Por Pressão Ministério da Saúde/Anvisa/Fiocruz. Brasília, 2013.

3. BRASIL. Portaria №. 529, de 1 de abril de 2013. Institui o Programa Nacional de Segurança do Paciente (PNSP). Brasília, 2013.

4. CARVALHO F, et al. Prevalência de lesão por pressão em pacientes internados em hospital privado do estado de Minas Gerais. Revista Enfermagem em Foco, 2019; 10 (4): 159-164.

5. COSTA A, et al. Custos do Tratamento de Úlceras Por Pressão em Unidade de Cuidados Prolongados em uma Instituição Hospitalar de Minas Gerais. Revista Enfermagem, 2018; 18:1.

6. DANTAS ALM, et al. Prevenção de Úlceras por Pressão Segundo a Perspectiva do Enfermeiro Intensivista. Revista de Enfermagem da UFPE online, 2019; 7(1): 706-12.

7. GALVÃO NS, et al. Aspectos epidemiológicos e clínicos de pacientes com úlcera por pressão internados em uma instituição hospitalar. Revista Estima, 2015; 13(3):sp.

8. GIRONDI J, et al. Lesão por pressão relacionada à dispositivos médicos: Revisão integrativa. Revista Enfermagem Atual In Derme, 2020; 93(31), p. e-020030.

9. MARTINS NBM, et al. Percepção de enfermeiros de terapia intensiva sobre prevenção de lesão por pressão. Revista de Atenção à Saúde, 2020; 18(63): 43-51.

10. MEDEIROS LNB, et al. Prevalência de Úlceras por Pressão em Unidades de Terapia. Revista de Enfermagem da UFPE online, 2017; (7): 2697-703.

11. MELLEIRO MM, et al. Indicadores de prevalência de úlcera por pressão e incidência de queda de paciente em hospitais de ensino do município de São Paulo. Revista Escola Enfermagem USP, 2015; 49(Esp2): 55-59.

12. MELO E, et al. Caracterização das Úlceras por Pressão em Pacientes de Unidade de Terapia Intensiva. Revista Estima, 2018; 12:3.

13. PEREIRA AFM, et al. Incidência de lesão por pressão em um hospital universitário. Revista de Enfermagem da UFPI, $2017 ; 6(1): 36-9$.

14. PETZ FFC, et al. Úlcera por pressão em unidade de terapia intensiva: estudo epidemiológico. Revista de Enfermagem da UFPE online, 2017; (Supl. 1): 287-95.

15. SANTOS ICRV, et al. Desbridamento Cirúrgico e a Competência Legal do Enfermeiro. Texto e Contexto Enfermagem, 2016; 22(1): 184-92.

16. SANTOS LRO, et al. Características demográficas e clínicas de pacientes de unidades de terapia intensiva com úlcera por pressão. Revista de Enfermagem da UFPE online, 2016; (Supl. 1): 225-31.

17. SILVA DRA, et al. Curativos de lesões por pressão em pacientes críticos: análise de custos. Revista da Escola de Enfermagem da USP, 2017; 51: e03231.

18. SIMAN AG, BRITO MJM. Mudanças na prática de enfermagem para melhorar a segurança do paciente. Revista Gaúcha de Enfermagem, 2016; (Supl.1):37.

19. SOARES EA, et al. Cultura de segurança do paciente e a prática de notificação de eventos adversos. Revista Acervo Saúde, 2019; 36, e1657.

20. SOBEST. Classificação das Lesões por Pressão - Consenso NPUAP 2016 - Adaptada Culturalmente para o Brasil. São Paulo, 2016.

21. SOUSA NCR. A implantação de uma escala de cicatrização de úlceras por pressão em um hospital de urgência e emergência em Teresina-PI. Trabalho de conclusão de curso (Pós-graduação em Enfermagem) - Centro de Ciências da Saúde. Universidade Federal de Santa Catarina, Florianópolis, 2017; 17p.

22. TEIXEIRA AKS, et al. Incidência De Lesões Por Pressão Em Unidade De Terapia Intensiva em Hospital com Acreditação. Revista Estima. 2017; 15(3): 152-160.

23. WORLD HEALTH ORGANIZATION (WHO). Conceptual framework for the international classification for patient safety version 1.1: final technical report. Geneva: WHO; 2010. 